\title{
An improved inequality related to Vizing's conjecture
}

\author{
Stephen Suen and Jennifer Tarr \\ University of South Florida \\ ssuen@usf.edu, jtarr2@mail.usf.edu
}

Submitted: Apr 7, 2010; Accepted: Dec 21, 2011; Published: Jan 6, 2012

Mathematics Subject Classification: 05C69

\begin{abstract}
Vizing conjectured in 1963 that $\gamma(G \square H) \geqslant \gamma(G) \gamma(H)$ for any graphs $G$ and $H$. A graph $G$ is said to satisfy Vizing's conjecture if the conjectured inequality holds for $G$ and any graph $H$. Vizing's conjecture has been proved for $\gamma(G) \leqslant 3$, and it is known to hold for other classes of graphs. Clark and Suen in 2000 showed that $\gamma(G \square H) \geqslant \frac{1}{2} \gamma(G) \gamma(H)$ for any graphs $G$ and $H$. We give a slight improvement of this inequality by tightening their arguments.
\end{abstract}

Keywords. Graph domination, Cartesian product, Vizing's conjecture

We use $V(G), E(G), \gamma(G)$, respectively, to denote the vertex set, edge set and domination number of the (simple) graph $G$. A $\gamma$-set of a graph $G$ is a dominating set of $G$ with minimum cardinality. For graphs $G$ and $H$, the Cartesian product $G \square H$ is the graph with vertex set $V(G) \times V(H)$ and two vertices are adjacent if and only if they are equal in one coordinate and adjacent in the other. In 1963, V. G. Vizing [4] conjectured that for any graphs $G$ and $H$,

$$
\gamma(G \square H) \geqslant \gamma(G) \gamma(H) .
$$

The reader is referred to Hartnell and Rall [3] and Brešar et al. [1] for a summary of the history and recent progress on Vizing's conjecture. Clark and Suen [2] in 2000 showed that for any graphs $G$ and $H$,

$$
\gamma(G \square H) \geqslant \frac{1}{2} \gamma(G) \gamma(H) .
$$

The following theorem is a slight improvement of this inequality.

Theorem 1. For any graphs $G$ and $H, \gamma(G \square H) \geqslant \frac{1}{2} \gamma(G) \gamma(H)+\frac{1}{2} \min \{\gamma(G), \gamma(H)\}$. 
Proof. Let $G$ and $H$ be arbitrary graphs, and let $D$ be a $\gamma$-set of the Cartesian product $G \square H$. Let $\left\{u_{1}, u_{2}, \ldots, u_{\gamma(G)}\right\}$ be a $\gamma$-set of $G$. We partition $V(G)$ into $\gamma(G)$ sets $\Pi_{1 .}, \Pi_{2 .}, \ldots, \Pi_{\gamma(G)}$., where $u_{i} \in \Pi_{i \text {. }}$ for all $i=1,2, \ldots, \gamma(G)$ and if $u \in \Pi_{i \text {. }}$ then $u=u_{i}$ or $\left\{u, u_{i}\right\} \in E(G)$.

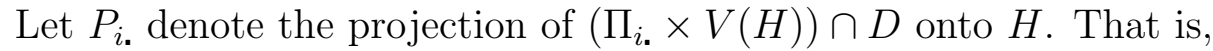

$$
P_{i .}=\left\{v \in V(H) \mid(u, v) \in D \text { for some } u \in \Pi_{i .}\right\} .
$$

Define $C_{i .}=V(H)-N_{H}\left[P_{i .}\right]$ as the complement of $N_{H}\left[P_{i .}\right]$, where $N_{H}[X]$ is the set of closed neighbors of $X$ in graph $H$. As $P_{i} \cup C_{i}$ is a dominating set of $H$, we have

$$
\left|P_{i .}\right|+\left|C_{i .}\right| \geqslant \gamma(H), \quad i=1,2, \ldots, \gamma(G) .
$$

For $v \in V(H)$, let

$$
D_{. v}=\{u \mid(u, v) \in D\} \quad \text { and } \quad S_{. v}=\left\{i \mid v \in C_{i .}\right\} .
$$

Observe that if $i \in S_{. v}$ then the vertices in $\Pi_{i .} \times\{v\}$ are dominated "horizontally" by vertices in $D_{. v} \times\{v\}$. Let $S_{H}$ be the number of pairs $(i, v)$ where $i=1,2, \ldots, \gamma(G)$ and $v \in C_{i .}$. Then obviously

$$
S_{H}=\sum_{v \in V(H)}\left|S_{. v}\right|=\sum_{i=1}^{\gamma(G)}\left|C_{i .}\right| .
$$

Since $D_{. v} \cup\left\{u_{i} \mid i \notin S_{. v}\right\}$ is a dominating set of $G$, we have

$$
\left|D_{. v}\right|+\left(\gamma(G)-\left|S_{. v}\right|\right) \geqslant \gamma(G),
$$

giving that

$$
\left|S_{. v}\right| \leqslant\left|D_{. v}\right| \cdot
$$

Summing over $v \in V(H)$, we have

$$
S_{H} \leqslant|D| .
$$

We now consider two cases based on (1).

Case 1. Assume $\left|P_{i .}\right|+\left|C_{i .}\right|>\gamma(H)$ for all $i=1, \ldots, \gamma(G)$. Then as $\left|\left(\Pi_{i .} \times V(H)\right) \cap D\right| \geqslant$ $\left|P_{i .}\right|$, we have

$$
\sum_{i=1}^{\gamma(G)}\left(\left|C_{i .}\right|+\left|\left(\Pi_{i .} \times V(H)\right) \cap D\right|\right) \geqslant \sum_{i=1}^{\gamma(G)}(\gamma(H)+1),
$$

which implies that

$$
S_{H}+|D| \geqslant \gamma(G) \gamma(H)+\gamma(G) .
$$

Combining (3) and (4) gives that

$$
\gamma(G \square H)=|D| \geqslant \frac{1}{2} \gamma(G) \gamma(H)+\frac{1}{2} \gamma(G) .
$$


Case 2. Assume $\left|P_{i .}\right|+\left|C_{i .}\right|=\gamma(H)$ for some $i=1, \ldots, \gamma(G)$. Note that $P_{i .} \cup C_{i \text {. is }}$ a $\gamma$-set of $H$. We now use this $\gamma$-set of $H$ to partition $V(H)$ in the same way as $V(G)$ is partitioned above. That is, label the vertices in $P_{i} \cup C_{i}$ as $v_{1}, v_{2}, \ldots, v_{\gamma(H)}$, and let $\left\{\Pi_{. j} \mid 1 \leqslant j \leqslant \gamma(H)\right\}$ be a partition of $H$ such that for all $j=1, \ldots, \gamma(H), v_{j} \in \Pi_{. j}$ and if $v \in \Pi_{. j}$, either $v=v_{j}$ or $\left\{v, v_{j}\right\} \in E(H)$. We next define the sets $P_{. j}, C_{. j}, S_{u .}$ and $D_{u .}$ in the same way $P_{i .}, C_{i .}, S_{. v}$ and $D_{. v}$ are defined above. To be specific, for $1 \leqslant j \leqslant \gamma(H)$, let

$$
P_{. j}=\left\{u \in V(G) \mid(u, v) \in D \text { for some } v \in \Pi_{. j}\right\}, \quad \text { and } \quad C_{. j}=V(G)-N_{G}\left[P_{. j}\right],
$$

and for $u \in V(G)$, let

$$
D_{u .}=\{v \mid(u, v) \in D\} \quad \text { and } \quad S_{u .}=\left\{j \mid u \in C_{. j}\right\} .
$$

Similarly, we have

$$
S_{G}=\sum_{u \in V(G)}\left|S_{u .}\right|=\sum_{j=1}^{\gamma(H)} C_{. j} .
$$

For $u \in V(G)$, let $\hat{D}_{u .}=\left\{v_{j} \mid\left(u, v_{j}\right) \in D_{u .}, 1 \leqslant j \leqslant \gamma(H)\right\}$. We claim that

$$
\left|S_{u .}\right| \leqslant\left|D_{u .}\right|-\left|\hat{D}_{u .}\right| .
$$

This is because $D_{u .} \cup\left\{v_{j} \mid j \notin S_{u .}\right\}$ is a dominating set of $H$, with

$$
D_{u .} \cap\left\{v_{j} \mid j \notin S_{u .}\right\}=\hat{D}_{u .},
$$

and the argument for proving (6) follows in the same way as (2) is proved. To make use of the claim, we note that when we partition the vertices of $H$, we have at least $\gamma(H)$ vertices in $D$ that are of the form $\left(u, v_{k}\right)$. Indeed, for each $k=1,2, \ldots, \gamma(H)$, either $v_{k} \in P_{i \text {. }}$, which implies $\left(u, v_{k}\right) \in D$ for some $u \in \Pi_{i .}$, or $v_{k} \in C_{i \text {. }}$, which implies that the vertices in $\Pi_{i .} \times\left\{v_{k}\right\}$ are dominated "horizontally" by some vertices $\left(u^{\prime}, v_{k}\right) \in D$. It therefore follows that

$$
\sum_{u \in V(G)}\left|\hat{D}_{u \cdot}\right| \geqslant \gamma(H)
$$

and hence summming both sides of (6)

$$
\sum_{u \in V(G)}\left|S_{u .}\right| \leqslant \sum_{u \in V(G)}\left(\left|D_{u .}\right|-\left|\hat{D}_{u .}\right|\right)
$$

gives that

$$
S_{G} \leqslant|D|-\gamma(H)
$$

To complete the proof, we note that similar to (1), we have

$$
\left|P_{. j}\right|+\left|C_{. j}\right| \geqslant \gamma(G), \quad j=1,2, \ldots, \gamma(H),
$$


and summing over $j$ gives that

$$
|D|+S_{G} \geqslant \gamma(G) \gamma(H) .
$$

Combining (7) and (8), we obtain

$$
\gamma(G \square H) \geqslant \frac{1}{2} \gamma(G) \gamma(H)+\frac{1}{2} \gamma(H) .
$$

As either (5) or (9) holds, it follows that

$$
\gamma(G \square H) \geqslant \frac{1}{2} \gamma(G) \gamma(H)+\frac{1}{2} \min \{\gamma(G), \gamma(H)\} .
$$

\section{References}

[1] B. Brešar, P. Dorbex, W. Goddard, B. L. Hartnell, M. A. Henning, S. Klavžar, and D. F. Rall, Vizing's Conjecture: A Survey and Recent Results, Journal of Graph Theory, 69, 46-76, 2012.

[2] W. E. Clark and S. Suen, An Inequality Related to Vizing's Conjecture, The Electron. J. of Combin. 7, Note 4, 2000.

[3] B. Hartnell and D. F. Rall, Domination in Cartesian Products: Vizing's Conjecture, in Domination in Graphs - Advanced Topics, edited by Haynes et al., 163-189, Marcel Dekker, Inc, New York, 1998.

[4] V. G. Vizing, The Cartesian product of graphs, Vyčisl. Sistemy 9, 30-43, 1963. 